\title{
Infini de Dieu et espace des hommes en peinture, conditions de possibilité pour la révolution scientifique $^{1}$
}

\section{Infinity of God and Space of Man in Painting, Conditions of Possibility for the Scientific Revolution}

\author{
Giuseppe Longo ${ }^{1}$, Sara Longo ${ }^{2}$ \\ ${ }^{1}$ CNRS, Ecole Normale Sup., Paris, \\ https://www.di.ens.fr/users/longo/ \\ ${ }^{2}$ History of Art, PhD, Paris 1, Panthéon-Sorbonne \\ https://hicsa.univ-paris1.fr/page.php? $r=23 \& i d=216 \& l a n g=f r$
}

RÉSUMÉ. Le riche débat grec et médiéval sur la nature de l'infini, en puissance et en acte, trouve une synthèse et une réponse dans l'invention, en Italie et à la Renaissance, de la perspective géométrique. Dès le milieu du XIV siècle, des peintres-théologiens donneront à l'infini en acte de Dieu sa première forme symbolique : la ligne ou le point de fuite dans les Annonciations, lieu de rencontre entre l'incommensurable et le mesurable, entre l'incirconscriptible et le lieu, entre Dieu et l'homme. La géométrie projective des peintres renaissants, une décision théologico-picturale, un geste à la limite infinie, organise alors l'espace des hommes, pour une humanité plus pleine, dans sa finitude, toujours confrontée à l'infini de Dieu. Ensuite, l'espace et l'infini encadreront les dynamiques physiques, en tant que conditions de possibilité pour la construction de la science moderne. Au cœur de cette nouvelle construction d'objectivité, le rôle du sujet connaissant et de la relativité des systèmes de référence est aussi à retrouver dans l'explicitation du regard relativisant du spectateur et de l'artiste en peinture. On posera la question de la pertinence de ses cadres, imprégnés d'a priori épistémiques, voire ontologiques, dans une science historique telle la biologie.

ABSTRACT. The rich Greek and medieval debate on the nature of the infinite, potential vs actual infinity, finds a synthesis and an answer in the invention, in Italy and during the Renaissance, of the geometric perspective. From the middle of the 14th century, painter-theologians gave actual infinite of God its first symbolic form: the line or vanishing point in the Annunciations, the meeting place between the immeasurable and the measurable, between the incircumscribable and the locality, between God and man. The projective geometry of the Renaissance painters, a theological-pictorial decision, a gesture at the infinite limit, organizes then the space of men, for a fuller humanity, in its finitude, yet always confronted with the infinity of God. Then space and infinity will frame the physical dynamics, as conditions of possibility for the construction of modern science. At the heart of this new construction of objectivity, the role of the knowing subject and the relativity of reference systems is also to be found in the explicitation of the relativizing gaze of the spectator and the artist in painting. The question will be raised as to the relevance of these frames, impregnated with epistemic, even ontological a priori, in a historical science such as biology.

MOTS-CLÉS. Perspective en peinture, Renaissance scientifique, infini mathématique, épistémologie, espaces physico-mathématiques, espaces biologiques.

KEYWORDS. Perspective in painting, scientific renaissance, mathematical infinity, epistemology, physicomathematical spaces, biological spaces.

\section{Une brève introduction à l'infini}

Il n'y a pas d'espace dans la géométrie grecque. En traçant des lignes, à la règle et au compas disons-nous aujourd'hui, on mesure, on construit des figures, sans un "contenant infini", un plan, un

${ }^{1}$ Dans “Les mathématiques dans l'oeuvre d'art" (R. Scheps et M.-C. Maurel resp.), ISTE-WILEY, 2020.

Version largement amplifiée et revue d'un texte paru dans ISTE OpenScience - Published by ISTE Ltd, London, UK, 2019, et, en forme très préliminaire en français, dans "Le formalisme en action : aspects mathématiques et philosophiques", (J.

Benoist, T. Paul eds) Hermann, 2013. 
espace, qui serait "derrière" elles. Des symétries - des rotations et des translations - produisent la preuve, au fini. Et l'infini potentiel (apeiron, sans limite, sans borne) est construit par des extensions, des itérations : on étend sans limite finie le segment en une droite (le deuxième axiome), eis apeiron. Si l'on se donne une collection de nombres premiers, on peut construire un nouveau nombre premier qui est plus grand que tout élément de cette collection (le théorème d'Euclide sur l'infinité des nombres premiers). Une extension et une itération sans fin du fini, du geste qui trace la droite à la construction des nombres entiers. Le temps est infini dans ce sens, jamais présent dans sa totalité dans notre pensée. L'infini n'est pas ce au-delà duquel il n'y a rien, dit Aristote dans La Physique, mais ce au-delà duquel il y a toujours quelque chose. Il est un devenir.

Paolo Zellini ${ }^{2}$ nous explique que la distinction aristotélicienne entre cet infini mathématique à construire pas à pas, potentiel, et l'infini qui est "déjà" là, en acte, qui englobe tout, sera reprise dans le débat métaphysique au cours du Moyen Âge. Dieu est un infini qui enveloppe tout, inclut tout, audelà duquel rien n'est donné. Mais ce concept d'infini actuel n'est pas simple. Il est compris par la négation par les aristotéliciens, comme chez Aristote; et Dieu ne peut pas avoir un attribut négatif. Saint Thomas toutefois forcera les esprits en excluant l'existence d'un tel infini en acte, sauf comme attribut de Dieu et lui seul. Et ce concept d'infini actuel se renforce, se précise positivement dans les esprits. Au point que l'évêque de Paris, Etienne Templier, décréta en 1277 l'infini actuel comme étant un attribut positif de Dieu et de sa création. Dieu, quand Il veut, installe l'infini actuel aussi dans le monde, par exemple en attribuant la Grâce Pleine et Infinie même à une femme, finie, Marie - le bûcher était prêt pour ceux qui n'étaient pas d'accord. Cette "posture axiomatique" ferme contribua certainement à stabiliser le concept d'infini.

Zellini souligne fort justement l'importance de ce débat pour la naissance d'une cosmologie de l'infini qui va trouver sa plénitude, mystique d'abord, puis scientifique, dans l'Univers infini et «gli infiniti mondi » de Nicolas de Cues (1401 - 1464) et Giordano Bruno (1548 - 1600).

\section{L'infini dans le tableau et l'invention de l'espace mathématique}

Le concept d'infini actuel se précise donc dans un débat métaphysique, qui cerne l'infini en une seule "entité" et force la pensée à le concevoir dans sa totalité. Comment va-t-il passer aux mathématiques, qui en feront un objet rigoureux du discours, voire une composante de la preuve ?

Le passage se fait par l'invention de la perspective (la « prospettiva ») dans la peinture italienne de la Renaissance ${ }^{3}$.

Le problème de la représentation des lieux où répartir les figures de la narration est au centre des attentions des peintres depuis la fin du XIII siècle. Les "boîtes" giottesques (ces maisons de poupée, sans un mur, ouvertes vers le spectateur) sont des lieux qui ont pour finalité de contenir l'historia et d'en rendre compréhensible l'enseignement théologique. Par un agencement contigu, des lieux spatiaux (boîtes, paysage, collines) rythment la narration - on y reviendra.

\footnotetext{
${ }^{2}$ P. Zellini, A brief history of infinity. New York: Penguin, 2005 (en italien : Adelphi, Roma, 1980).

${ }^{3}$ En Italie, le débat fut aigu à l'époque sur le nom à attribuer à cette nouvelle technique : " perspettiva ", un "voir à travers", qui passera aux autres langues, ou, de façon plus pertinente, le choix d'un point de vue, une " prospettiva ", comme on le verra.
} 


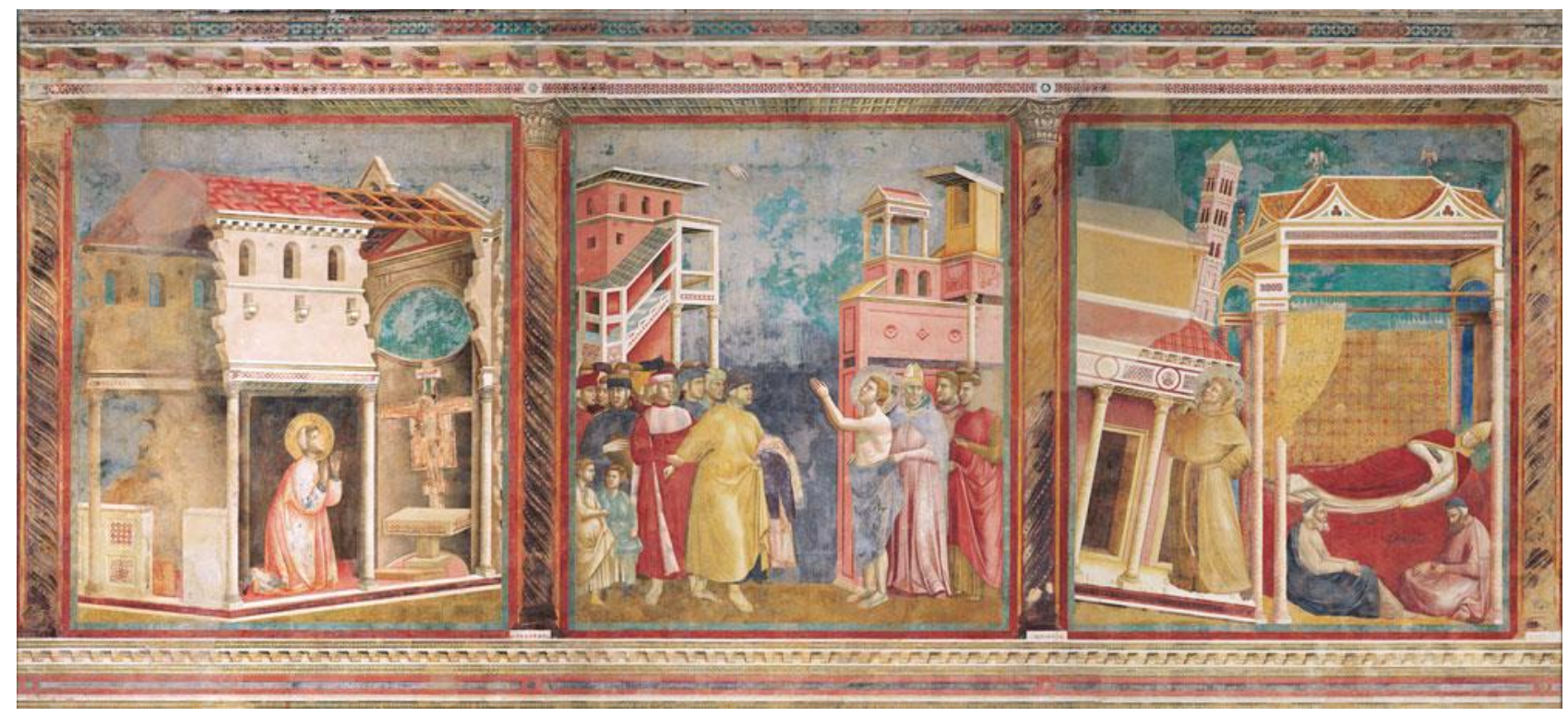

Giotto di Bondone, Vie de saint François, fresque, vers 1290. Assise, basilique saint François.

La perspective géométrique expérimentée par Filippo Brunelleschi en 1417 et définie en 1435 par Leon Battista Alberti est une révolution: non seulement elle construit un espace unique de la composition (et donc, sauf quelques rares exceptions, une narration unifiée) mais surtout elle est le fait d'une construction où l'homme est à l'origine de toute mesure (voir Alberti, De pictura, I, 19) et où l'infini, point de convergence des lignes orthogonales à la base du tableau, est contenu, enfermé dans le cadre de la représentation. Depuis la seconde moitié du siècle dernier et en réaction à l'article inaugural d'Erwin Panofsky (La perspective comme forme symbolique, 1925), des historiens de l'art comme Pierre Francastel, Hubert Damisch et Louis Marin à l'École des Hautes Études en Sciences Sociales, ont mis en évidence l'importance de cette révolution picturale.

Dans l'essai fondateur d'Erwin Panofsky, publié en Allemagne en 1927 mais traduit en français seulement en 1975, la représentation d'un espace par la géométrie des lignes orthogonales développerait «le concept d'un infini dont il n'y a pas seulement un modèle en Dieu, mais qui est effectivement réalisé dans la réalité empirique $»{ }^{4}$. Erwin Panofsky a noté que l'Annonciation d'Ambrogio Lorenzetti (ci-dessous), peinte un siècle avant la théorisation d'Alberti, est la première construction géométrique où les fuyantes convergent, non pas vers un seul point, mais vers un seul axe vertical (sur le plan du tableau, la colonne qui sépare Gabriel et Marie). Daniel Arasse ira plus loin pour étendre cette intuition à l'essor tout à fait particulier de constructions géométriques complexes dans les scènes de l'Annonce à Marie.

Son argumentation est tout à fait pertinente pour notre propos : 1'affinité particulière qui a existé, au $X^{\mathrm{e}}$ siècle, entre Annonciation et perspective, est due au fait que, dans l'histoire chrétienne, le moment où l'infini vient dans le fini et bien celui de la venue miraculeuse du fils de Dieu dans la chair humaine, par la rencontre entre Dieu et la Madone, pleine de Grâce. Daniel Arasse discute cette idée par la mise en évidence de ce qu'il appelle un souci d'ordre «théologico-pictural », qui joue des effets et de l'efficace de l'image: les allers-retours entre profondeur et surface, les paradoxes internes aux structures spatiales de certaines Annonciations, manifestent l'impossibilité de figurer Dieu dans l'espace de la géométrie humaine. Cette recherche en peinture serait intimement liée à une conception du divin qui n'est pas exclu, comme disait Panofsky, mais présent dans le tableau.

\footnotetext{
${ }^{4}$ Erwin Panofsky, La perspective comme forme symbolique, p. 157.
} 
Pour appuyer son propos, Arasse s'appuie, en particulier, sur un sermon de Saint Bernardin de Sienne prononcé sur le campo de Sienne en 1427: 1'Annonciation est le moment où " l'immensité vient dans la mesure [...], l'infigurable dans la figure, l'incirconscriptible dans le lieu, l'invisible dans la vision [...], la longueur dans la brièveté, la largeur dans l'étroitesse, la hauteur dans la bassesse $»^{5}$... autant de paradoxes conceptuels qui sont à l'origine de paradoxes spatiaux de la part des peintres. Daniel Arasse met aussi en évidence comment les plus savants perspectivistes se plaisent à se jouer des règles de la perspective géométrique pour montrer la venue paradoxale de l'infini dans le fini.

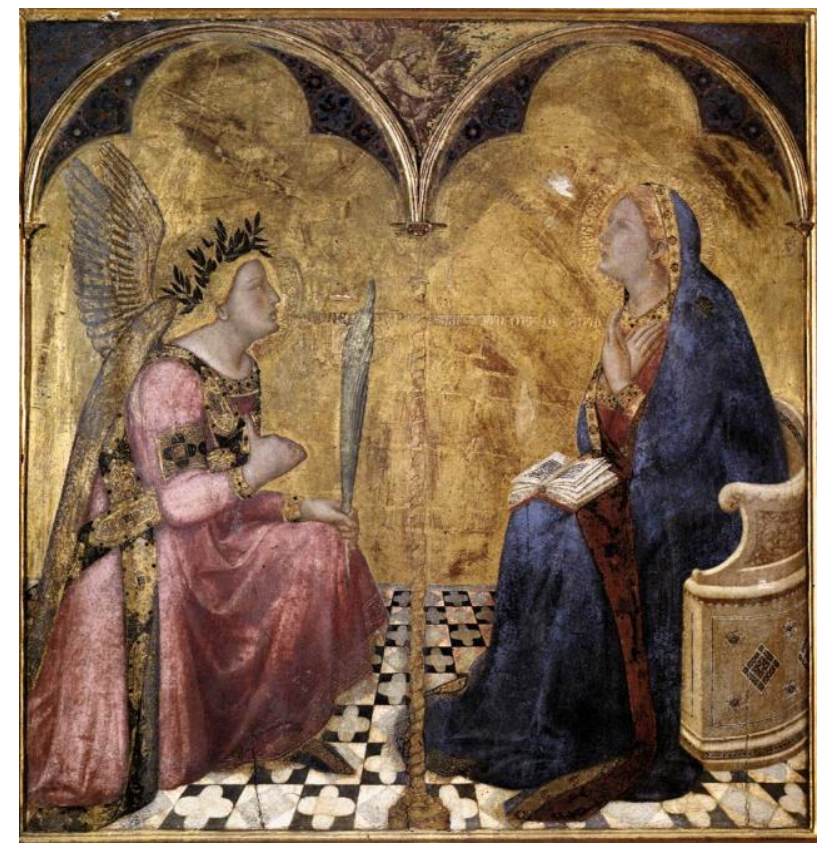

Ambrogio Lorenzetti, Annonciation, détrempe sur bois, 1344. Sienne, Pinacoteca nazionale.

Dans cette Annonciation, une colonne, souvent un symbole du Christ, bien concrète au sol, s'atténue vers le haut où elle se superpose et cache l'axe de fuite, disions-nous, à l'infini, une référence explicite à Dieu. Voilà une extraordinaire nouveauté, en 1344 : un espace projectif dessiné avec rigueur. Et, du coup, par l'effet de la géométrie de ce plancher qui va de l'homme à Dieu, un nouveau lieu se déploie : Dieu y a sa place, caché, lointain à l'infini, mais présent dans l'histoire racontée. Et la Madone aussi a une nouvelle épaisseur humaine : son corps solide, tridimensionnel, accompagne l'expression d'un humanisme qui se dessine. La perspective introduit Dieu comme limite actuelle, à l'infini, donc comme la limite d'un espace que tout englobe, y compris les espaces humains qui se renouvellent. Et les tous premiers tableaux peints en «prospettiva » seront des annonciations, ce lieu unique de la rencontre infini/fini ${ }^{6}$. Ensuite, avec Piero della Francesca, cet exposé en peinture d'une métaphysique deviendra aussi une technique, sans nécessairement perdre son âme religieuse. Le livre de Piero, De prospectiva pingendi, est un vrai traité de géométrie projective "pratique", le plus important texte de mathématiques de son époque, écrira Vasari.

Or, la "prospettiva'" permet au peintre d'organiser l'espace des hommes et des choses ainsi que de choisir un point de vue. Le choix de l'emplacement du point de fuite fixe le point de vue du

\footnotetext{
${ }^{5}$ Saint Bernardin de Sienne, De triplici Christi nativitate, dans Opera omnia, Venise, 1745, IV, p. 3, cité dans San Bernardino de Siena, Pagine scelte, Milan, 1950, p. 54.

${ }^{6}$ Sara Longo, "L'Annonciation en Italie. Enjeux méthodologiques et historiographiques, autour du colloque florentin de 1986 », " 'La perspective de l'Annonciation', présentation d'une étude de Daniel Arasse » et "L'intervalle sacré » in Studiolo, revue de l'Académie de France à Rome, X, 2013, p. 24-32 et p. 75-93.
} 
spectateur, il propose/impose un regard, comme celui qui voit, humblement d'en bas, le saint Sébastien martyr d'Antonello da Messina (1476).

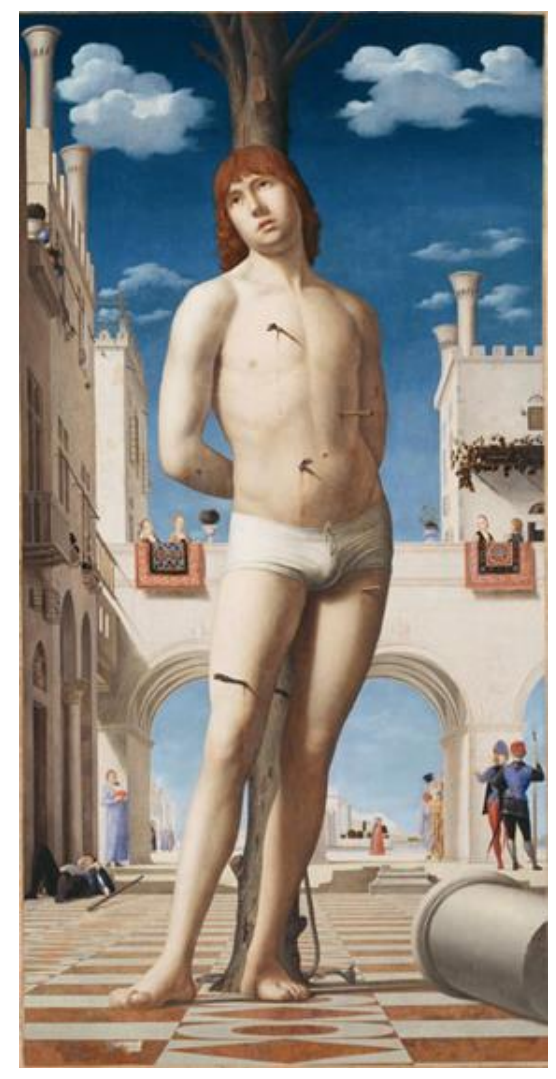

Antonello da Messina, Saint Sébastien, détrempe sur bois transposée sur toile, 1476.

Dresde, Gemäldegalerie.

Et voilà que cette métaphysique et cosmologie religieuse devient une géométrie de l'espace : Dieu, les astres et les hommes y trouveront un nouvel emplacement, organisé par un point de vue unifiant, et modifiable. On est bien loin des absolus hors de l'espace et du monde des mosaïques byzantines, que l'on se souvienne de Ravenne. Cette (ré-)organisation de l'espace, ce talent nouveau à choisir un point de vue, cette technique bientôt répandue en Europe aidera Copernic (1473 - 1543) à "voir" le système solaire du "point de vue du Soleil", la nouvelle "prospettiva" de la science moderne".

Dans l'invention de la perspective renaissante, c'est donc bien de l'invention simultanée d'une structure explicite de l'espace et d'un point de vue qu'il s'agit, et même d'une 'révolution copernicienne" de ce point, nouveau et relativisant, on y reviendra en sciences. Elle est la conséquence d'un changement diamétral : le spectateur ne doit plus se déplacer selon un système d'agencement de lieux contigus, il est le point fixe à partir duquel se déroule un récit continu. Le changement est très important, car il situe différemment l'homme dans l'espace de l'action voire dans l'Univers : l'espace physique aristotélicien résulte de la somme de lieux différents, il n'a pas de structure globale et donne forme à un espace pictural conçu comme la somme des lieux de l'historia. La plus haute expression de cette idée étant les boîtes spatiales de Giotto, disions-nous, et de ses compagnons du Trecento : des "maisonnettes", voire des pièces, sont habitées par les acteurs de différentes histoires, souvent indépendantes. La construction monofocale théorisée par Alberti en 1435, quatre-vingt-dix ans après les premières expériences de Lorenzetti, bouleverse cette pratique. En faisant de l'espace pictural un corps unifié, bien qu'articulé en lieux (ses membres), elle aura

\footnotetext{
${ }^{7}$ Il s'agit d'une remarque dans B. C. van FRASSEN, An introduction to the Philosophy of Space and Time, Random House, New York, 1970. 
pour particularité d'être construite à partir d'un point unique et mathématique, proposé par le peintre et qui identifie aussi l'œil du spectateur immobile, relativement auquel tout s'organise.

L'idée du point de vue unique et immobile, choisi, constitue un saut théorique, une mutation épistémologique ${ }^{8}$. La peinture pense l'espace jusqu'alors fermé du tableau comme ouvert sur le récit « presque jusqu'à une distance infinie " ${ }^{9}$. Comme nous l'ont appris, entre autres, Hubert Damisch et Daniel Arasse, la peinture pense mais, plus encore, elle montre. La perspective linéaire, la costruzione leggittima albertienne, donc, est une abstraction géométrique qui unifie les lieux de l'histoire et en cela elle constitue une rupture théorique par rapport aux techniques précédentes. Et l'espace mathématique est posé.

\section{L'optique géométrique et le sujet dans l'espace projectif}

Les peintres et les scientifiques du $\mathrm{XV}^{\mathrm{e}}$ paraissent bien connaître l'optique géométrique, inventée par Euclide et développée par les grandes écoles de l'algèbre et géométrie de langue arabe. Certains auteurs soulignent les références chez Alberti aux "anciens"' qui avaient si bien étudié les réflexions sur les surfaces et les convergences des rayons de lumière ${ }^{10}$. Bien évidemment, il s'agissait d'une analyse des lignes au fini, parfois poussées "eis apeiron", sans limite, comme chez Euclide, donc potentiellement vers l'infini, par exemple dans les calculs des angles qui sous-tendent un objet qui s'éloigne de l'œil. Idn al-Haytham (Egypte, $\mathrm{XI}^{\mathrm{e}}$ siècle) a été l'un des grands maîtres de ces calculs. Mais l'infini actuel, décision métaphysique difficile, n'était pas là, ce point projectif à la limite asymptotique qui structure l'espace et force un point de vue pour tout spectateur.

L'optique géométrique interviendra toutefois massivement dans le travail des peintres, à partir du $\mathrm{XV}^{\mathrm{e}}$ siècle ; elle introduira une nouvelle rigueur géométrique, enrichira le répertoire figuratif pour mieux représenter les distances et organiser les figures dans l'espace projectif. Mais elle n'est pas à l'origine du tournant métaphysique, antérieur d'un siècle, qui a permis l'invention picturale de l'espace que nous évoquons ici.

Concernant cette nouvelle rigueur technique, dans un article rarement cité, Daniel Arasse prend en exemple les tavolette de Brunelleschi, considérées comme le premier exemple d'une géométrie rigoureuse dans la construction "prospettica'": il décrit l'action de l'ingénieur/architecte florentin lors de la vérification de sa représentation en perspective du Baptistère et propose au lecteur d'inverser les étapes de la vérification telles qu'elles sont racontées par Antonio Manetti dans la biographie de Brunelleschi.

Brunelleschi trace le baptistère vu depuis l'entrée de Santa Maria del Fiore. Il fait ensuite un trou à la hauteur du point de fuite et retourne la tablette avec le dessin. Il met son œil face au trou et regarde le vrai baptistère. Puis il soulève un miroir à la hauteur du dessin et regarde le reflet ; en baissant puis levant à nouveau le miroir, il vérifie la conformité entre ce qu'il voit devant lui et les lignes de son desin ${ }^{11}$.

\footnotetext{
${ }^{8}$ Micheal Baxandall a très finement mis en relation la conception albertienne de la peinture avec le modèle oratoire de la rhétorique. Voir M. BAXANDALL, Giotto et les humanistes : la découverte de la composition en peinture, 1340-1450, Paris, Points, 2013 (Clarendon Press 1971).

${ }^{9}$ L. B. ALBERTI, La peinture, 2004, p. 84.

${ }^{10} \mathrm{G}$. SIMON, Optique et perspective : Ptolémée, Alhazen, Alberti. Revue d'histoire des sciences, 54, n³, $2001 . \mathrm{pp} .325-350$.

${ }^{11}$ A. MANETTI, Opere historiche, Florence, G. Milanesi, 1887, p. 83-85.
} 


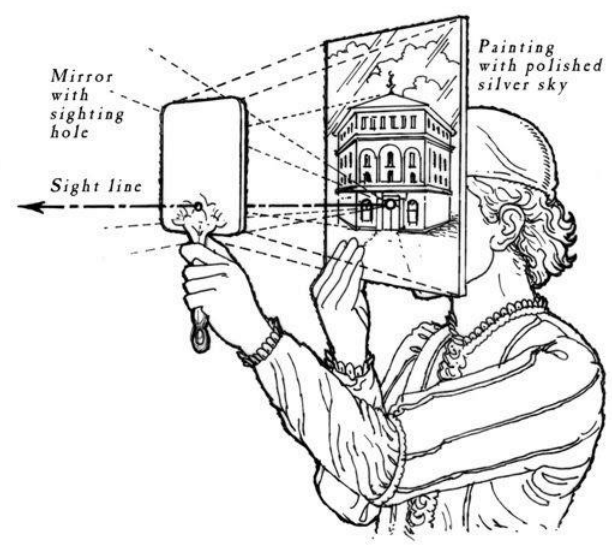

Daniel Arasse imagine le sujet qui se regarde d'abord dans le miroir, puis qui lève le bras avec la tavoletta retournée entre lui-même et le miroir, appliquant un seul œil au trou pratiqué dans la tablette pour voir le dessin. Le reflet de celui qui se regarde s'occulte au profit du reflet du Baptistère puis, en baissant le miroir, au profit de la vision du vrai bâtiment. «... Le modèle théorique de la peinture en perspective se constitue par l'occultation de l'image du moi dans le miroir, à laquelle est substituée l'image de l'ensemble du visible $»^{12}$.

Cette idée de renverser les modalités d'exécution d'un procédé de vérification élevé par l'histoire de l'art au rang de paradigme cognitif permet de rapprocher le récit de la vérification de Brunelleschi à l'autre mythe fondateur de la peinture, celui de Narcisse, dont le visage s'abolit dans la source. Mais dans notre cas, le sujet revient. Car le point de vue est toujours choisi par le peintre, le choix du placement de l'horizon est la première opération conseillée par Alberti. Ainsi, à partir des années 1450, les horizons placés particulièrement bas ou haut traduisent une conscience précise de la présence d'un regardeur, que les effets de plongée ou de contre-plongée vont frapper. Par le choix du point projectif, la position supposée du regardeur conditionne l'espace de la peinture. « Ce qui se marque là, c'est que l'image n'existe pas sans celui qui l'a élaborée ${ }^{13}$. Le sujet moderne, peintre, spectateur, est inventé ${ }^{14}$.

On retrouve ces inventions sous le pinceau de l'autre grand «perspecteur », Andrea Mantegna, cette fois dans un corps peint. Pas n'importe lequel, celui du Rédempteur, à peine déposé de la croix, non pas dans son tombeau mais sur un plan de marbre. Par un sublime raccourci perspectif, nous sommes placés au plus près des pieds du Christ et le regardons non pas da sotto in su, mais des pieds à la tête. Le choix de Mantegna est audacieux, au point que l'on s'accorde à voir dans le Christ mort le testament artistique du padouan.

Pour mieux comprendre le tableau regardons d'abord des études. Parmi des dessins récemment attribués à Mantegna et publiés, on retrouve la recherche du perspecteur, amoureux des corps représentés en raccourci, tout comme dans les dessins préparatoires pour le Christ mort: le dessin étudie le corps du Christ vu depuis les pieds puis depuis la tête, mais toujours légèrement de biais par rapport au plan de la représentation, et non pas à quatre-vingt-dix degrés, comme dans le tableau final.

\footnotetext{
${ }^{12}$ D. ARASSE, « La peinture de la Renaissance italienne et les perspectives du Moi », 1983, p. 237.

${ }^{13}$ D. ARASSE, « La peinture de la Renaissance italienne et les perspectives du Moi », 1983, p. 238.

${ }^{14}$ Sara LONGO, thèse de Doctorat en Histoire de l'art, "Voir et savoirs dans la théorie de l'art de Daniel Arasse", en cours de publication, Paris 1, Panthéon-Sorbonne, 2014.
} 


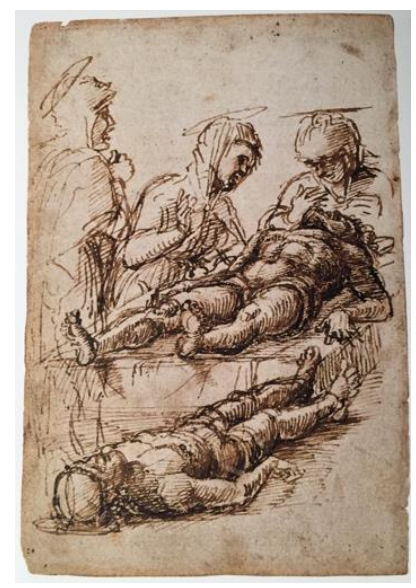

Andrea Mantegna, Étude pour le Christ mort, plume et lavis brun sur papier, 1460-1465. Milan, collection privée.

L'exercice est le même chez Paolo Uccello, dans les mêmes années. Lorsque sa femme l'appelait dans la nuit, nous raconte Vasari, Paolo la rejoignait au lit en marmonnant : «Oh ! quelle douce chose que cette perspective !» ... Les corps allongés de la bataille de San Romano donnent au sol de la bataille toute sa profondeur.

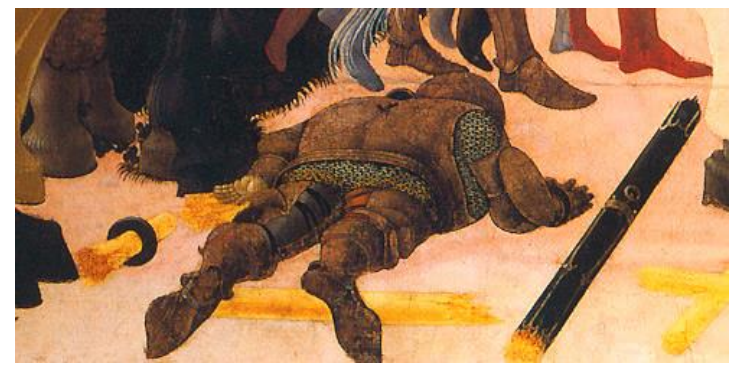

Paolo Uccello, Bataille de San Romano, détail, détrempe sur bois, 1456. Londres, National Gallery.

Mais l'invention retenue dans le Christ mort est unique : le point de vue choisi est celui du pénitent, du fidèle en adoration qui se penche sur les stigmates - celui de Madeleine peut-être.

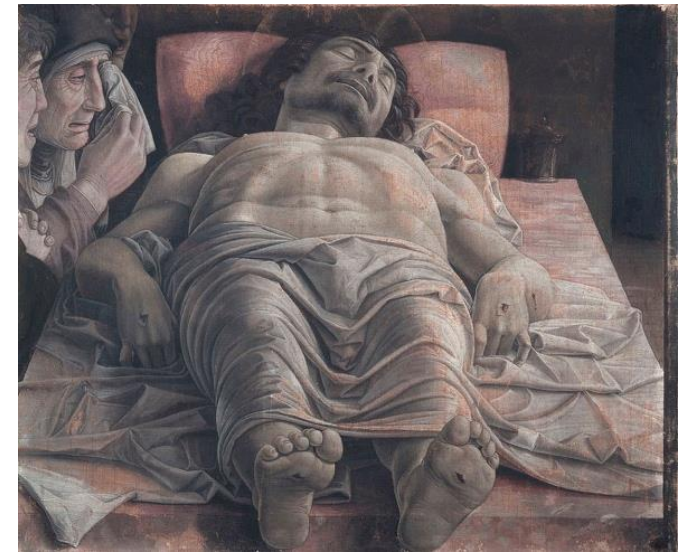

Andrea Mantegna, Christ mort, détrempe sur toile, 1490. Milan, Pinacoteca di Brera.

Son flacon d'onguent est posé sur le marbre près de la tête du Christ. Celle-ci repose sur un coussin, lui aussi en marbre rose, pour ne pas déroger au chromatisme minéral de l'ensemble du tableau (corps, drap et lit, tout est de pierre). À gauche, trois figures pleurent le corps mort. En bas, c'est nous. Nous regardons ce corps et le peintre a bien pris soin de tout nous montrer : les plantes de pieds et les dos des mains écorchés par les clous, les malléoles latérales et le péroné puis, sur le 
torse, le nombril, le stigmate du flanc droit et la poitrine. Enfin, sur le visage, menton, bouche, nez, yeux, sourcils, front et cheveux, tout est parfaitement visible et dénombrable.

Un raccourci perspectif rigoureux et géométrique issu d'un simple calcul depuis un point de vue n'aurait pas permis cela. Le front aurait été caché par le nez, le nez par le menton, le torse par le ventre et, surtout, les pieds auraient été immensément plus grands. La linéarité de la perspective et l'optique géométrique sont consciemment dépassées, par décision du peintre. On peut dire, en positivant, que le point de vue n'est pas exactement à la hauteur des pieds, que nous sommes un peu surélevés et la tête du Christ est aussi levée, sur le coussin de marbre. Or l'impression de raccourci est bien là, nous sommes aux pieds du Christ, mais notre adoration porte sur tout son corps, et c'est donc tout le corps que le peintre doit donner à voir. Aucun réalisme là-dedans, comme le confirment les bras, qui sont reliés au buste par d'étranges et impossibles muscles qui partent de sous la poitrine. Anatomiquement, le corps est monstrueux; picturalement, il est parfaitement en monstration. En bref, la perspective, en apparence et en principe si rigoureuse, n'a rien d'absolu, encore moins d'objectif. Elle est un relatif par lequel le sujet regardant façonne la forme.

Un dernier exemple, encore une Annonciation, peinte par Piero della Francesca autour de 1470 pour les sœurs tertiaires de l'ordre de saint Antoine de Pérouse.
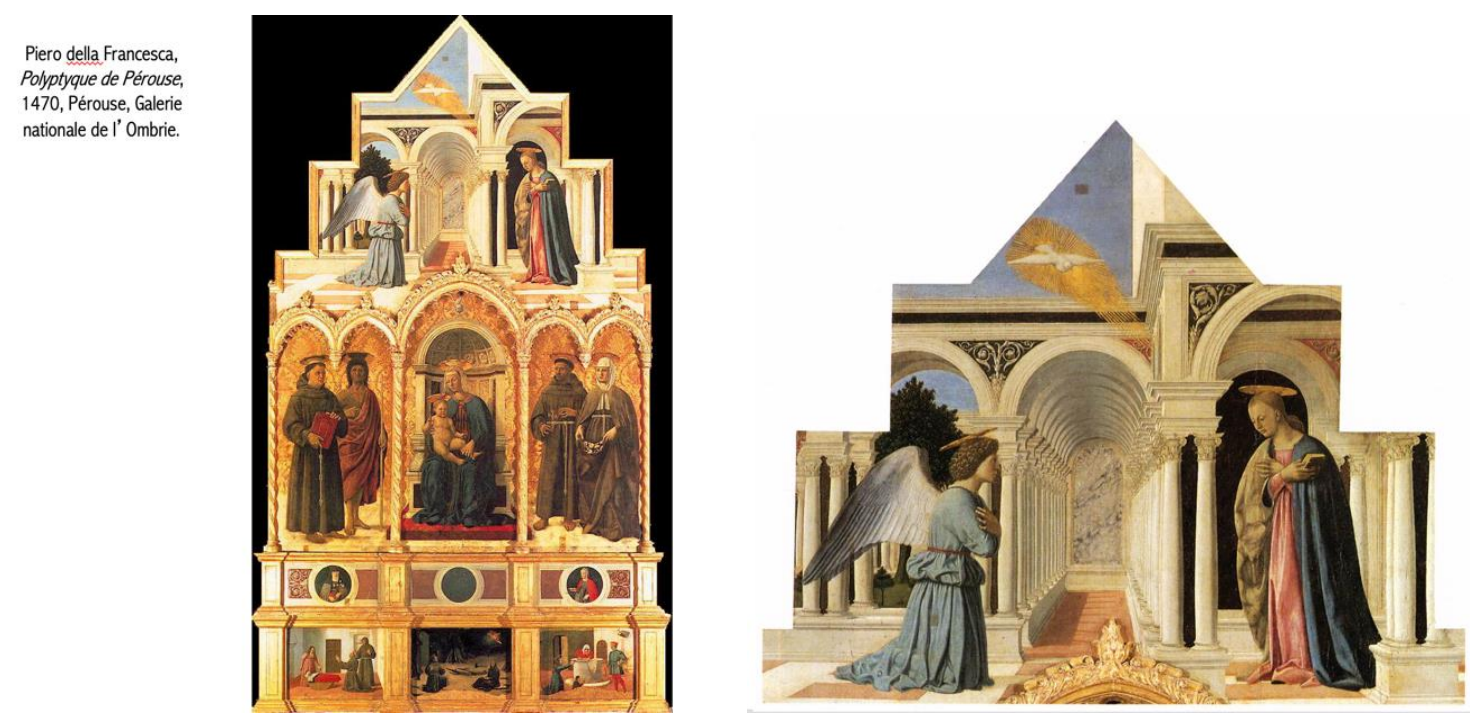

Polyptyque de Pérouse, registre supérieur (voir plus haut).

L'Annonciation qui décore la cimaise de ce grand retable est peinte dans un espace que le cadre ne clôt pas. La perspective permet en effet à Piero de donner l'illusion que ce que nous voyons, découpé par l'imposant cadre à degrés, n'est qu'une partie d'un espace bien plus ample, celui d'un cloître. Contrairement au registre médian, où le fond doré donne une unité spatiale mais peu de profondeur, et au registre inférieur, prédelle traditionnelle où chaque scénette a son propre espace de narration perspectif, unifié et fermé, le registre supérieur donne à voir un espace infiniment grand, autant sur les côtés (chaque élément architectural sort du cadre), qu'en profondeur (l'enfilade de colonnes est impressionnante). Gabriel est agenouillé devant Marie et lève les yeux vers elle. Marie croise les mains et baisse légèrement la tête en signe d'acceptation. Elle n'est pas devant l'architecture, comme Gabriel, mais sous une avancée du bâtiment. Que le lecteur regarde les massifs des colonnes à droite et à gauche de la figure de Marie : ils sont les seuls éléments du tableau situés au premier plan. Or la Vierge est bien face à Gabriel, à la même distance de nous, on le voit à leurs pieds posés sur la même bande blanche de pavement, qui est derrière ces soubassements. Quel est l'effet visuel de cette disposition des personnages dans l'architecture ?

Il y a moins d'un demi-siècle, des historiens de l'art amoureux de perspective ont remarqué un paradoxe au sein de la grande complexité géométrique de cette peinture. Du point de vue de Gabriel, 
Marie est invisible. Elle est cachée par le deuxième massif de colonnes qui ne nous est pas visible, à nous spectateur, car nous sommes presque à quatre-vingt-dix degrés de l'enfilade... mais ce massif doit être là, puisque, face à Marie, en haut, on distingue bien deux arcs. C'est donc vers des colonnes qu'elle s'incline, et non vers Gabriel. Thomas Martone, qui a relevé et discuté cette invention, emploie la belle formule de «trompe-l'intelligence ${ }^{15}$. Piero della Francesca, peintre géomètre par excellence, joue des paradoxes impliqués par un double point de vue, le nôtre et celui de l'ange, pour tromper non pas notre œil mais notre esprit.

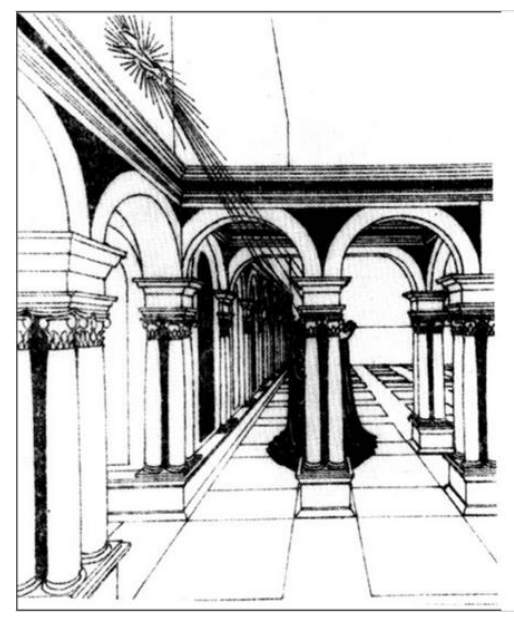

De Martone, article cité.

Nous, fidèles en prière, voyons Gabriel et Marie face à face et contemplons leur dialogue, leur échange spirituel qui devient le support de notre prière, de notre croyance. Nous devons penser qu'ils se voient pour dialoguer. Mais, géométriquement, rationnellement, nous dit (en secret) Piero, les deux ne peuvent se voir, car ils se voient en pensée, leur dialogue est muet et aveugle. Théologiquement, il y a entre eux le divin, le miracle dans toute son invisibilité qui n'est pas explicable par la rationalité, encore moins géométrique, mais qui est rendu possible par la finesse de l'organisation géométrique de l'espace. Car la peinture, au Quattrocento, n'est pas seulement conçue comme la reproduction de «tout ce qui se voit sous le soleil » et dont la « fin est la délectation » (Poussin, 1665, lettre à son ami Frédéric de Chambray). Elle est aussi support d'invisible, de foi dans un mystère que les lignes et les couleurs ne sont pas là pour expliquer mais, par des jeux de visibilités, pour montrer qu'il y a secret ${ }^{16}$. Le paradoxe émerge de l'emploi si rigoureux d'une géométrie qui permette de situer les protagonistes dans une situation théologiquement choisie, et ce loin de tout réalisme naïf. Forcé par ce paradoxe, le spectateur est investi du double rôle visuel et spirituel ; l'objectivité géométrique est au service de l'échange des regards entre les protagonistes, des points de vue, de ce qui est montré et caché au spectateur.

Ce jeu entre relatif et objectif, ancré dans le rapport à l'espace du sujet regardant/connaissant, dans l'explicitation d'un point de vue subjectif pour permettre la construction de l'objectivité, sera au cœur de la science moderne, jusqu'à nos jours, comme nous essayerons de mettre en évidence ${ }^{17}$. La forme picturale de l'infini en acte participe à l'invention de cet espace et du sujet scientifique.

\footnotetext{
${ }^{15}$ T. MARTONE, « Piero della Francesca e la prospettiva dell'intelletto », in Piero teorico dell'arte, Gangemi, 1985, p. 173-185. Voir aussi Jean PETITOT, Morphologie et Esthétique, Paris, Maisonneuve et Larose, 2004, p. 75-84.

${ }^{16}$ Sur la question du secret montré comme caché, voir les remarques de Louis Marin, Opacité de la peinture : essais sur la représentation au Quattrocento, Paris, Usher, 1989.

${ }^{17}$ On pourrait ici reprendre la remarque de Van Frassen au sujet de Copernic dans la note plus haut : prendre le point de vue du Soleil pour regarder le système solaire et le jeu de cache-cache des planètes et de leurs satellites n'est point "réaliste".
} 
Explorons maintenant différents articulations, mathématiques et pratiques, de cet infini et de ces espaces.

\section{4 - La limite du temps, le calcul et l’algèbre}

Revenons d'abord aux enjeux de l'infini. Le temps, depuis Aristote, est considéré comme un infini potentiel paradigmatique, car jamais présent dans sa totalité dans notre pensée. Quel sens "temporel" aurait sa limite projective ${ }^{18}$ ? Par contre, il est intéressant de voir cette arrivée, au sein des mathématiques, d'une première expression symbolique d'infini actuel, constitué dans un débat religieux, qui se fait en passant par la peinture. Une peinture explicitement mathématisée, en particulier par Piero della Francesca, disions-nous, peintre et géomètre. Cela peut se comprendre en observant que la construction conceptuelle et géométrique d'un espace infini ne s'appuie pas nécessairement sur un infini actuel : l'espace de Descartes peut très bien être conçu comme un infini potentiel, une extension sans fin à partir de son origine. L'infini actuel, par contre, est inhérent à la construction projective, en particulier quand elle est utilisée pour la représentation bidimensionnelle d'un espace tridimensionnel. Et le point de fuite rend objectif l'infini actuel ; il le montre, là-bas, au fond du tableau. Bref, à la Renaissance, suite au rôle central de la peinture dans l'art et dans la construction de la connaissance (pensons aux dessins de Léonard de Vinci), y compris théologique, on a pensé l'espace tridimensionnel mathématiquement, en le dessinant d'abord; pour le rendre intelligible (visible) sur un plan, il a fallu alors le projeter, en montrant ainsi une forme (symbolique) de cet infini de Dieu, en acte, si bien défini par les philosophes chrétiens du Moyen Âge.

Deux siècles plus tard, Desargues rendra pleinement mathématique cette synthèse projective entre infini et géométrie. En Analyse, Newton et Leibniz proposeront les notions de dérivé et intégrale comme opérations à limite infinie, au cœur du calcul différentiel. Cantor, au XIX ${ }^{\mathrm{e}}$ siècle, objectivera ultérieurement, par une syntaxe, l'infini actuel, en lui associant une nouvelle expression symbolique, scripturale cette fois, $\omega$. En le maniant algébriquement, il en engendrera une infinité, $\omega+1, \omega+2 \ldots$, jusqu'à inventer une arithmétique des infinis, $\omega+\omega, \omega \times \omega \ldots \omega^{2}, \omega^{3}, \ldots \omega^{\omega} \ldots$, les ordinaux transfinis. Rien ne stabilise un concept mieux qu'une praxis mathématique, qu'un usage technique d'un signe pour le concept, dont le sens s'enrichira par ce même usage. Le débat sur l'infini de Dieu concernera aussi Cantor, un homme profondément religieux : Dieu sera (à) la limite de toutes les limites, audelà de ses transfinis ${ }^{19}$.

Il faut observer que, dans tous ces cas, l'infini mathématique nous donne un outil pour l'intelligibilité du monde. Dans la peinture de la Renaissance, la géométrie projective, cette décision théologique, ce geste asymptotique, à l'infini, organise l'espace des hommes, pour une humanité plus pleine, dans sa finitude. De façon similaire, des espaces de Descartes et Desargues à l'infini actuel de Newton et Leibniz, la physique-mathématique rendra intelligible le mouvement au fini, autour de nous, par des infinis, ceux du calcul infinitésimal. En logique, à partir de Gentzen (1935),

\footnotetext{
${ }^{18}$ Dans la tentative de rendre compatible la Relativité avec la théorie du Big-bang, certains physiciens pensent à l’origine du temps comme limite inverse asymptotique, c'est-à-dire à l'inverse de l'ordre temporel donné par l'expansion de l'Univers. En fait, les théorèmes de Noether (la conservation d'énergie comme invariant des équations du mouvement par translations temporelles) sont au cœur du tournant relativiste et incompatibles avec une origine du temps, voir F. Bailly, G. Longo, Mathematics and Natural Sciences : the Physical Singularity of Life, Imperial College Press, London, 2011 (Traduction et révision du livre pour Hermann, Paris, 2006).

${ }^{19}$ N. Charaud, Georg Cantor, Infini et inconscient, Spartacus IDH, 2019 : https://spartacus-idh.com/collections/nouvelles-visionsdes-sciences.html\#
} 
l'analyse ordinale de la preuve se basera sur l'arithmétique des infinis de Cantor ${ }^{20}$. Du reste, déjà Galilée expliquait à Simplicius, dans l'analyse d'une sphère mathématique s'appuyant sur un plan par un seul point (insensé, dit Simplicius), que les mathématiques sont une science à la limite infinie $^{21}$. Le fini fétichise l'itération et il en reste prisonnier ${ }^{22}$. Le formaliste finitiste qui rejette l'infini en le déclarant hors du monde et de la physique, ne saisit pas le sens humain, pour nos dess(e)ins et pour nos praxis d'intelligence, de ce geste qui pose l'infini dans le monde, en organisant ce même monde par le langage, la géométrie et l'écriture.

Une épistémologie de ce concept organisateur ne peut être qu'historique : une histoire des idées et des praxis constitutives, une histoire critique, toujours à repenser.

\section{Les espaces rationnels : du commerce à la physique}

La naissance de la science moderne se fait donc par la construction d'un espace infini des possibles, un espace et un temps de tout phénomène et de toute dynamique physique possible. Le choix de l'origine des coordonnées cartésiennes propose le système de référence dans lequel décrire toutes les trajectoires possibles et dans lequel se construira la physique, après Galilée. En fait, l'approche analytique de Descartes pose l'origine et la mesure de l'espace, elle lui donne une "prospettiva", à partir de laquelle on pourra encadrer et comprendre le monde. En tant qu'invariant de la représentation mathématique, cet espace de toutes les courbes, de toutes les trajectoires est certainement le constitué d'une pluralité d'actes d'expérience. La cartographie des nouvelles explorations géographiques, l'optique arabe ${ }^{23}$ contribueront, au XVI ${ }^{\mathrm{e}}$ siècle, à mieux expliciter, du point de vue mathématique, ce nouvel encadrement du monde. En particulier, Francesco Maurolico (1494 - 1575) développa une synthèse remarquable des idées des grands géomètres et algébristes de l'Islam, un aspect du mariage fécond des traditions occidentale et orientale en mathématiques ${ }^{24}$.

Dans ce contexte, l'innovante et riche relation galiléenne entre expérience et théorie, la théorisation physique elle-même, a pour objet de rendre intelligibles et prévisibles les phénomènes : on observe d'abord, on prend des mesures et, par la suite, la théorie devrait engendrer une prédiction permettant de la vérifier. L'anticipation scientifique, en fait mathématique, du futur est alors placée au centre de l'intelligibilité du monde proposée par la science moderne. Et cette prédiction s'effectue dans l'espace et le temps des événements physiques, décrits mathématiquement par la représentation analytique cartésienne, enrichie par celle que l'on appelle la "relativité galiléenne" : l'espace-temps moderne des phénomènes physiques est né d'une analyse du passage d'un système de référence (cartésien), un point de vue, à un autre, tout en préservant les lois de la physique. Plus précisément, dans l'espace-temps infini des trajectoires possibles, donné a priori, les invariants sont décrits en termes de symétries - le groupe des transformations de Galilée entre systèmes de référence en

\footnotetext{
${ }^{20}$ L'infini entre dans la preuve, en fait, comme infini entre algèbre et géométrie, celui du "bon ordre" des entiers, G. Longo "Reflections on Concrete Incompleteness", Philosophia Mathematica 19(3): 255-280, 2011 (les articles dont G. Longo est (co-) auteur sont téléchargeables de https://www.di.ens.fr/users/longo/download.html )

${ }^{21}$ Une histoire qui est un point de départ de A. Gargani, /l sapere senza fondamenti, Einaudi, 1975.

${ }^{22}$ G. Châtelet, Les enjeux du mobile, Seuil, Paris, 1993.

${ }^{23}$ E. Kheirandish, "The Manāẓir Tradition through Persian Sources," Les sciences dans le monde iranien, ed. Ž. Vesel, et al., Tehran, 1998, repr. 2004, pp. 125-45. E. Kheirandish, ed. and tr., The Arabic Version of Euclid's Optics: Kitāb Uqlīdis fï ikhtilāf al-manāẓir, 2 vols., New York, 1999.
}

${ }^{24}$ A. Islami, G. Longo, 2017. Marriages of Mathematics and Physics: a challenge for Biology. In The Necessary Western Conjunction to the Eastern Philosophy of Exploring the Nature of Mind and Life (K. Matsuno et al., eds), Special Issue, Progress in Biophysics and Molecular Biology, Vol 131, 179-192. 
mouvement relatif rectiligne uniforme (inertiel). Voilà donc l'objectivité de la loi scientifique donnée par la relativité des systèmes de référence, les transformations possibles de l'un dans l'autre : le relatif permet l'objectif. C'est le sujet toutefois qui choisit ces systèmes de référence, s'y situe, fixe un point de vue parmi d'autres, comme le faisait le peintre, en le posant pour le spectateur. La certitude scientifique de la loi (ce qui est invariant) permet alors la prédiction, le calcul, justifie la théorie.

Il faudrait toutefois situer le basculement vers le mythe de la prédiction rationnelle d'un avenir scientifiquement prévisible dans l'espace des possibles, comme s'étant, lui aussi, opéré au début de la Renaissance italienne. L'audace de se doter d'un aperçu de l'avenir par des moyens rationnels et à l'intérieur d'un espace de possibilités donné, remonte à l'appréciation du progrès et à la capacité d'en faire des estimés quantitatives, en Italie aux XIVe et XVe siècles. C'est alors que les technologies des artisans, voire des grandes structures productives (l'« arsenale di Venezia », dont parlera Galilée) commencèrent à changer le rapport au travail et à la transformation de la Nature elle-même ${ }^{25}$. Et c'est alors que l'on inventa le crédit bancaire, à l'époque du tableau de Lorenzetti, dans cette même Toscane ${ }^{26}$. Les mathématiques interviendront ensuite massivement dans ce progrès : Luca Pacioli (1445 - 1517), en particulier, avec la Summa de Arithmetica, Geometria et autres écrits, développe considérablement l'arithmétique de Fibonacci da Pisa (1170 - 1250) et invente la «partita doppia », un outil fondamental pour la finance et le commerce.

Bref, vers la moitié du $\mathrm{XIV}^{\mathrm{e}}$ siècle, en Italie, le prêt monétaire était désormais permis, en particulier sous la forme de «lettres de crédit» ou de monnaie-papier. Puisqu'il ne s'agissait plus d'un péché, il était alors possible de miser sur les progrès futurs, d'obtenir de l'argent d'une banque, puis d'investir et d'anticiper le retour de l'argent investi avec intérêts calculables et, donc, d'en faire un gain personnel, ce qui était révolutionnaire sur les plans économique et conceptuel. Il n'y avait plus aucune nécessité de magie, ni de divination dans l'anticipation du progrès et dans la capacité de prédiction de l'avenir ; il s'agit plutôt de connaissance rationnelle, voire mathématique. Bien sûr, les aléas étaient possibles, mais à l'intérieur d'un espace de possibilités prédéfini : comme lorsque l'on lance un dé, on prend un risque, mais parmi les six résultats possibles, ni plus ni moins - et les symétries du dé nous donnent les probabilités. Les attentes et les paris sont rationnels : on peut évaluer le risque, dans un espace pré-donné de la finance, Pacioli, et des dés (G. Cardano "De ludo alea”' 1560, G. Galilée “'Sopra le scoperte dei dadi'”1612).

Et ainsi naît la société du progrès futur anticipé parmi une liste prédéterminée de dynamiques possibles. La société dans laquelle l'on peut oser emprunter et prêter de l'argent ainsi que construire la connaissance scientifique à l'intérieur d'un espace des trajectoires futures mathématiquement prédéfini, quoique infini ; une science où il est possible de prédire, grâce à une théorie scientifique, le résultat d'une expérience, voire d'une activité économique ou commerciale. La peinture avait rendu géométriquement possibles, nous avons vu, ces espaces de l'action des hommes par une contamination vertigineuse avec l'infinité de Dieu. Et l'objectivité était déjà atteinte en explicitant la relativité du point de vue, en mettant en évidence le rôle du sujet qui regarde, ce spectateur point neutre dans son rapport à l'espace, au point de savoir même "forcer' le voir, au-delà, voire contre la linéarité de la costruzione leggittima, comme chez un grand maître de la perspective, Mantegna.

\footnotetext{
${ }^{25}$ Rossi P. 1962. I filosofi e le macchine, Feltrinelli, (ristampa 2002).

${ }^{26}$ Le prêt de la Banque Bardi et Peruzzi au roi de Grande-Bretagne Edward III date de 1332. II n'a jamais été rendu - comme le savent très bien tous les lycéens italiens et notre collègue Alberto Peruzzi à Florence.
} 


\section{Se donner à priori les conditions de la représentation et du savoir}

La révolution inspirée de la pratique picturale de la perspective et sa théorisation, est résumée en 1500 dans le De Sculptura de Pomponio Gaurico : avant de peindre un objet, le peintre doit construire le cadre spatial (le locus) pour le situer - le "locus" précède les corps qui l'habitent. Panofsky se réfère explicitement à Gaurico pour souligner la naissance d'une vision kantienne de l'espace, comme a priori ou "condition de possibilité" pour faire de la science ${ }^{27}$.

Après Newton, Kant posera donc l'espace et le temps comme conditions de possibilité, a priori de la construction scientifique : techniquement, pour écrire les équations et résoudre les équations, il faut tout d'abord se donner tous les paramètres et les grandeurs pertinentes.

Lagrange et Laplace, au XVIII ${ }^{\mathrm{e}}$ siècle, donneront ensuite les mathématiques des "systèmes à états déterminés" modernes. En effet, en résolvant des équations de Newton dans les espaces de Descartes et Galilée, « on doit pouvoir prédire tous les faits de la mécanique » (de la mécanique céleste, disait Laplace mais il visait en fait à l'ensemble des faits de la physique). L'analyse mathématique des probabilités de Pascal et de Laplace traite également l'imprévisibilité comme aléatoire, mais un aléatoire qui est n'a rien à voir avec la détermination, à analyser en termes de probabilité, donc d'une mesure sur un espaces de tous les possibles. Car, dans tous les cas, pour eux, les événements imprévisibles ont lieu à l'intérieur de l'espace infini mais prédéterminé, cartésien, de toutes les trajectoires et de tous les faits possibles. Cet espace sera généralisé, au XIX siècle, à l'espace des phases, c'est à dire, à l'espace des observables et paramètres pertinents : à l'énergie on associera le temps, à l'impulsion la position. Voilà donc un parcours possible qui, de l'espace unifié dans lequel a lieu la rencontre entre l'infini de Dieu et le fini d'une femme, souvent une chambre, une cour, nous conduit aux espaces très généraux de la physique mathématique contemporaine. La structuration rigoureuse, infinitaire, de cette chambre, de cette cour, en est un passage historique fondamental.

Dans ces espaces mathématiques, Poincaré, à la fin du XIX ${ }^{\mathrm{e}}$ siècle, intégrera l'imprévisible à la détermination, en nous montrant l'imprédictibilité de dynamiques parfaitement déterministes. Bien différemment, la mécanique quantique introduira l'aléatoire dans la théorie, sous la forme d'indéterminisme intrinsèque. Toutefois, les espaces des "trajectoires" et des événements possibles, des phases, demeureront toujours mathématiquement prédéfinissables, qu'ils soient infinis, de Descartes à Poincaré, ou qu'il s'agisse même d'espaces de dimension infinie - les espaces de Hilbert ou de Fock de la mécanique quantique. Dans ces espaces, la trajectoire d'une loi de probabilité, déterminé par l'équation de Schrödinger, hors de l'espace-temps ordinaire, déterminera les dynamiques quantiques; la mesure, en projetant sur un nombre réel cette dynamique d'une probabilité, rendra le caractère indéterministe de la mécanique quantique. Par conséquent, cette théorie aussi donne a priori les espaces des évolutions possibles, qui peuvent accommoder l'événement quantique le plus imprévisible, dont la création/annihilation d'un quanton. Au $\mathrm{XX}^{\mathrm{e}}$ siècle, comme dans les inventions perspectives de la renaissance, le sujet rentrera à nouveau et massivement dans la construction scientifique : le quanton est le résultat de l'interaction entre le sujet, son regard par l'instrument de mesure, et le "réel"' microphysique - il se forme et déforme, il se constitue, sous ce regard. Et, sous des formes nouvelles, le sujet connaissant revient.

\footnotetext{
${ }^{27}$ Une vaste synthèse du débat sur la naissance du concept moderne d'espace et le rôle de la perspective, de Gaurico à Panofsky, peut être trouvée dans V. De Risi "Arte e scienza della sfera. La nascita del concetto moderno di spazio fra la teoria rinascimentale della prospettiva e la geometria di Leibniz" Sphaera : Forma immagine e metafora, tra Medioevo ed Età Moderna, Olschki, Roma, 2012. D'accord avec Damisch et Arasse, nous avons souligné le jeu entre théologie et humanisme qui en est à l'origine.
} 
Notons, et c'est important, que la description finie de ces espaces infinis, des espaces cartésiens aux espaces quantiques aux dimensions infinies, est rendue possible par leurs régularités : elles sont données en termes de symétries mathématiques, c'est-à-dire en tant qu'ensembles d'invariants et de transformations préservant ces invariants. Les symétries permettent la synthèse finie, voire formelle et axiomatique, des espaces infinis, même de dimension infinie ; le hasard, même quantique, y sera mathématiquement encadré, il restera à l'intérieur de l'espace prédéfini des possibles.

Notons enfin que, dans la richesse des explorations des peintres renaissants, on entrevoit un paradoxe inversé du hasard et de l'espace, parfaitement irréaliste, dans un des tableaux dont nous avons vu un détail, l'une des trois Batailles de San Romano de Paolo Uccello. Les "axes cartésiens" du plan sont données par les lances et hallebardes brisées qui, tombées au hasard du chaos de la bataille, jonchent le sol de façon parfaitement orthogonale, engendrant ainsi l'espace.

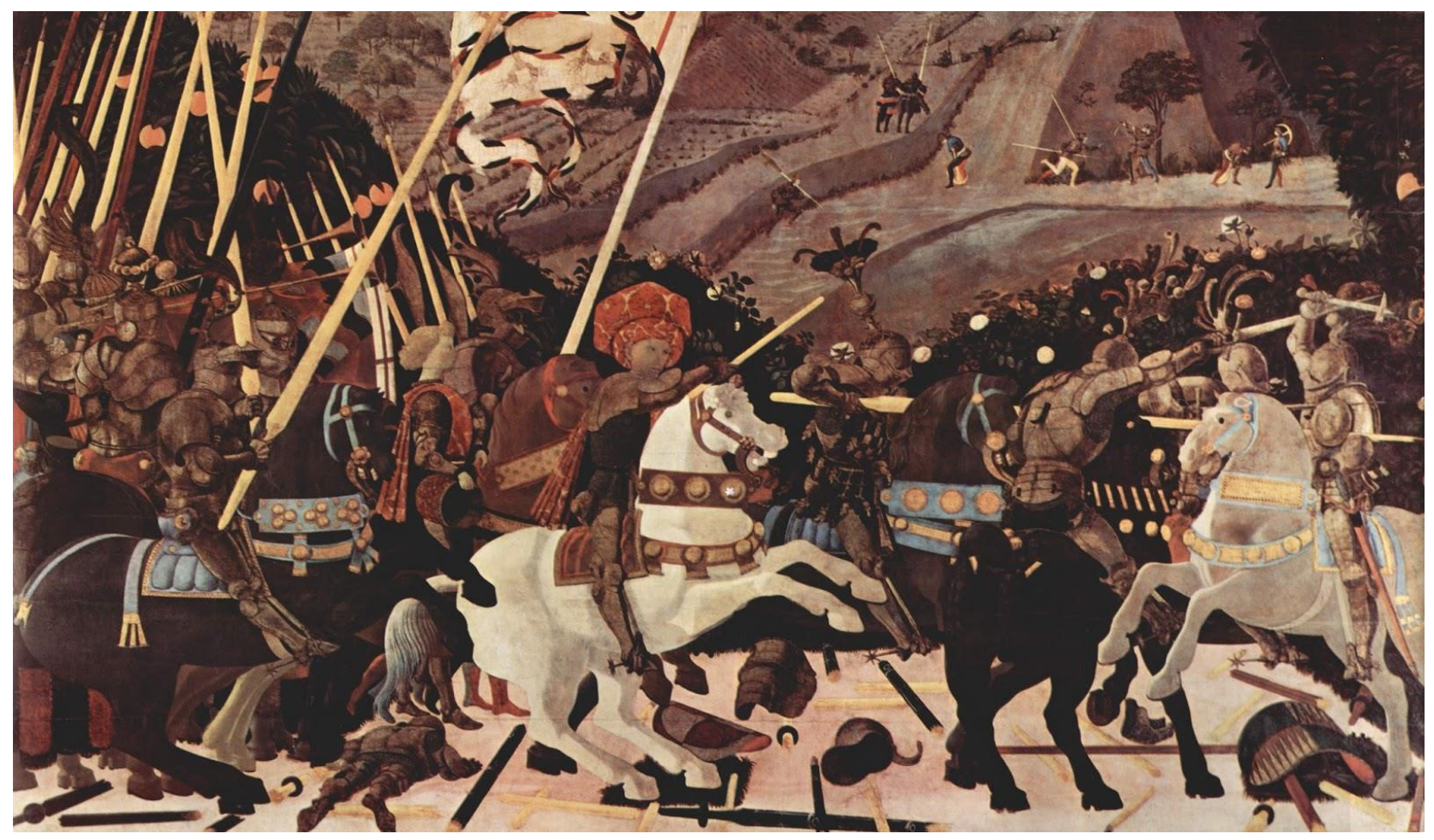

Paolo Uccello, Bataille de San Romano, détrempe sur bois, 1456. Londres, National Gallery.

En conclusion, dans ce texte nous avons essayé de reconstruire un parcours constitutif de la puissance des mathématiques des espaces infinis de tous les possibles de la physique, même quand ces possibles sont donnés en termes de probabilités (quantiques), à partir d'une origine théologique qui trouve dans la peinture du $\mathrm{XIV}^{\mathrm{e}}$ siècle sa première expression symbolique.

\section{Quels espaces des possibles pour l'évolution du vivant ?}

C'est là où nous avons aujourd'hui un problème : dans l'analyse des phénomènes du vivant, comme phénomènes biologiques mais aussi sociétaux, il n'y a aucune manière de prédéterminer (mathématiquement) l'espace même des évolutions possibles, des "phases" du vivant. Essayons de préciser davantage cette idée. L'aléatoire du lancer d'un dé ou d'une pièce de monnaie, ou encore, d'un événement quantique, opère, comme l'on disait plus haut, dans un espace prédéfini des dynamiques possibles. Nous savons nous donner des infinis mathématiques, depuis Lorenzetti et Piero della Francesca, depuis Descartes, Desargues, Newton, Cantor et Hilbert. Leurs symétries (les invariants mathématiques) permettent de définir géométriquement et formellement ces espaces (des phases, des possibles). 
En revanche, il n'y a aucune façon de prédéterminer l'espace des phénotypes (formes biologiques) futurs possibles au cours de l'évolution - et les phénotypes, voire les organismes, sont les observables biologiquement pertinents. D'aucune façon n'y avait-il un signe du nez des mammifères dans les "formes", ni dans l'ADN bactériens d'il y a 800 millions d'années. Ni pouvaiton mettre dans une liste de possible les os internes de leur oreille, comme dérivés de la double mâchoire de quelques vertébrés (les gnathostomes) d'il y a 250 millions d'années (un exemple de "exaptation", adaptation ex-post ${ }^{28}$ ) : tout phénotype est le résultat d'une histoire, d'un vaste réseau génétique et d'interactions épigénétiques évolutives, changeants.

Mais même la liste des événements biologiques possibles durant le prochain siècle, par exemple les formes des eucaryotes, ne se trouve pas dans des espaces mathématiquement prédéfinissables : au cours de l'évolution, les phénotypes et les écosystèmes se co-constituent et produisent conjointement l'espace des possibilités, à partir des niches que chaque organisme construit dans et avec son environnement. Et des moindres fluctuations dans ces interactions, à l'intérieur, voire entre les différents niveaux d'organisation, ne changent pas seulement des "trajectoires" dans des espaces de phases pre-donnés, comme dans les dynamiques physiques, mais ces mêmes espaces. Les symétries, c'est à dire les transformations qui définissent l'invariance et qui régissaient magnifiquement la physique se trouvent continuellement modifiées, car la biologie est

\section{l'itération jamais identique d'un processus morphogénétique,}

à partir de la reproduction cellulaire et jusqu'à la morphogenèse la plus complexe, qui simultanément co-déterminent la structure de l'écosystème. La stabilité structurelle préserve certaines symétries globales (p. ex. les plans d'organisation de base ou bauplan), mais chaque mitose constitue un changement de symétrie, brise l'invariance physico-mathématique : les deux nouvelles cellules ne sont jamais identiques, pas même à la cellule mère. Ce "jamais identique", une diversité du protéome, de l'ADN, des membranes, de la chromatine ..., la reconstitution de la matrice d'un tissue, après une mitose dans un multicellulaire, est un changement de symétries.

Et cela est fondamental pour qu'il y ait la variabilité et la diversité qui permettent l'évolution et l'ontogenèse. La diversité de chaque embryogenèse, moindre ou importante (le "hopeful monster" de Goldschmidt qui peut en résulter), participe de la production des changements évolutifs. Car ces changements permanents (de symétries), en particulier au niveau des observables pertinents, les phénotypes, sont au cœur de la variabilité, donc de la diversité et de la possibilité même de l'état vivant de la matière. Ils permettent la sélection parmi les nouveautés ainsi que l'adaptabilité et contribuent aux modifications de l'écosystème.

Bref, pour nous

la trajectoire phylogénétique et ontogénétique d'un organisme est une cascade de changements de symétries ${ }^{29}$.

Bien évidemment, les analyses exclusivement moléculaires, voire genocentrées, dont les observables sont bien pré-définissables, sont intrinsèquement incomplètes, quoique très utiles : elles n'arrivent même pas à décrire la transmission héréditaire des certaines déformations acquises de la

\footnotetext{
${ }^{28}$ Gould S.-J. 2002. The Structure of evolutionary Theory, Harvard U. Press.

${ }^{29}$ Pour un cadre général dans cette perspective, voir Soto A., Longo G., Noble D. (eds.) 2016. From the century of the genome to the century of the organism: New theoretical approaches, a Special issue of Progress in Biophysics and Mol. Biology, Vol. 122, 1, Elsevier.
} 
membrane des ciliés, voire leurs structures lipidiques ${ }^{30}$, ni les dynamiques du protéome lors des modifications induites et héritées de l'operon lactose - même donc au niveau, dans ce cas, purement moléculaire.

Les mathématiques constituent une science des invariants et des transformations préservant les invariants, et donc une science des symétries. Serons-nous en mesure d'inventer de nouvelles mathématiques pour traiter les changements continuels des symétries ? Pourquoi pas ? Les pères fondateurs ont bien inventé leurs outils, les mathématiques de l'invariance, d'Euclide à Newton et Riemann et Grothendieck - sa notion de "topos", en tant que notion d'espace catégorique, très générale, est le maximum actuel d'une philosophie et une pratique des mathématiques en tant que science des invariants et des transformations qui les préservent - le parcours a été long depuis Lorenzetti, mais ce dernier a contribué à l'ouvrir. Dans tous les cas, nous devons oser l'invention nouvelle, afin d'aborder le vivant ainsi que l'économie, bien à l'écart des absurdes théories de l'équilibre dans des espaces pré-donnés : il n'existe pas d'écosystème ni de système économique à l'état d'équilibre, ni tendant vers l'équilibre, aux géodésiques uniques et pré-données, à moins que tous les "agents" ne soient morts. Le vivant n'est pas seulement un processus bien loin de l'équilibre, il est toujours en "transitions", sur un seuil critique : du point de vue mathématique, il est (dans) une "transition critique étendue" (voir Bailly, Longo, cité). Comme l'économie, il est toujours "en crise".

\section{Conclusion et ouverture : les espaces hétérogènes de l'évolution biologique}

Nous avons essayé d'esquisser un fil conducteur possible, à travers l'histoire, de la notion mathématique d'espace, si profondément mêlée à celle d'infini. L'explicitation du rôle du sujet connaissant en fait aussi partie, dans un jeu complexe avec la construction "relativisante", de l'objectivité scientifique. L'origine théologico-picturale de l'espace physico-mathématique moderne lui donne des racines particulièrement profondes et en fait un a priori qui semble échapper à la critique scientifique. Son passage de la théologie aux mathématiques par le biais de la peinture est la thèse que l'on propose ici. Or, une épistémologie qui s'enrichit d'une histoire possible peut aider à changer de regard et de pratique, quant à la constitution de nos espaces de connaissance.

Le défi de la dynamique changeante de l'espace des possibles se pose dans toute science historique, disions-nous. En particulier, rien en biologie ne peut être compris si ce n'est à la lumière d'une perspective temporelle, d'une historicité propre, celle de l'évolution ${ }^{31}$. Or cette histoire se caractérise par l'émergence de nouveaux "espaces de phases" ou, plus précisément, de nouveaux observables, les phénotypes et les organismes darwiniens. Nous avons mentionné l'émergence de l'oreille interne des vertébrés; parmi les maintes exemples, on pourrait en citer un autre qui concerne les “dimensions du temps" comme outil d'intelligibilité : les rythmes biologiques, comme ceux de la respiration, des battements cardiaques ..., se sont constitués au cours de l'évolution et sont différents des fréquences physiques. Ces rythmes sont mieux compris en les inscrivant dans une

\footnotetext{
${ }^{30}$ Cavalier-Smith, T. 2004 The membranome and membrane heredity in development and evolution. In Organelles, genomes and eukaryote phylogeny: an evolutionary synthesis in the age of genomics (eds R. P. Hirt \& D. S. Horner), pp. 335-351. Boca Raton, FL: CRC Press

${ }^{31}$ Dobzhansky, T 1973. Nothing in Biology Makes Sense Except in the Light of Evolution, American Biology Teacher, 35 (3): $125-$ 129. Pour un regard recent: Montévil, M. 2020. Historicity at the hearth of biology. Theory in Biosciences, to appear.
} 
deuxième dimension temporelle, en proposant donc un nouvel observable et son propre espace mathématique ${ }^{32}$.

Trois livres et plusieurs articles écrits en collaboration avec Francis Bailly, Maël Montévil et, en co-direction, avec Ana Soto (voir http://www.di.ens.fr/users/longo/) esquissent de nouvelles structures conceptuelles visant à mieux comprendre la singularité physique de l'état vivant de la matière : le changement de perspective sur les symétries est au cœur d'une telle proposition scientifique. La pensée d'un espace des possibles pré-donné, invariant donc, une symétrie fondamentale de la physique, où, depuis Lorenzetti, on arrive même à inscrire Dieu, ne suffit plus. La prévisibilité, même celle de l'espace des possibilités, n'est plus au centre de la construction de la connaissance. Cette construction vise la compréhension de la contingence historique du vivant (et, éventuellement, de la société - mais nous ne poussons pas si loin nos analyses). Cela ne doit pas entraver la conscience du rôle de notre action dans un monde foncièrement imprévisible, sans espace qui le sous-tende, dans lequel nous jugeons pour le mieux, en rendant explicite la perspective (et les valeurs) guidant nos actions.

Bref, le défi théorique est ainsi d'inventer les outils visant à comprendre mais non pas nécessairement à prédire, disait déjà René Thom. La théorie de l'évolution de Darwin ne prédit rien, mais elle nous fournit un extraordinaire cadre de connaissance. Les estimés qualitatifs portant sur les effets d'une activité peuvent nous permettre d'agir dans le monde, si ces estimés sont fondés sur des critères de robustesse de développement tels que la diversité et la capacité d'adaptation.

La science est une de nos formes actives d'être au monde, entre connaissance et praxis. Nous construisons la connaissance aussi pour agir dans ce monde et nous avons effectivement besoin de prévisibilité, mais il est possible que celle-ci soit provisoire et qualitative, que l'on soit obligé de sortir du mythe de ces possibles pré-donnés aussi beau et tranquillisant que les espaces de Piero, tout en gardant le cap de l'intelligibilité, qui permet l'action sensée. Des tentatives de type théorique ${ }^{33}$ et mathématique sont en cours, comme celles des groupes Cardano ${ }^{34}$ et des "Dynamiques poststructurelles" ${ }^{35}$, centrées sur un nouveau calcul différentiel de l'hétérogenèse - un calcul dont les opérateurs engendrent leurs propres espaces des possibles ${ }^{36}$. L'inspiration de la sémiotique et le dialogue avec l'art contemporain y est explicite.

\footnotetext{
${ }^{32}$ Longo G. 2020. Confusing biological twins and atomic clocks. Today's ecological relevance of Bergson-Einstein debate on time. Conference "What is time? Einstein and Bergson 100 years later", April 4-6, 2019 (proceedings in preparation, Campo, Ronchi eds).

33 Longo, G. 2020 Naturalizing Physics. Or, embedding physics in the historicity and materiality of the living. Deleuziana, n. 11, special issue on "Differential Heterogenesis: Deleuze, Mathematics And The Creation Of Forms".

${ }^{34}$ http://cardano.visions-des-sciences.eu/fr

${ }^{35}$ https://enseignements-2019.ehess.fr/2019/ue/2691/
}

${ }^{36}$ Sarti, A., Citti, G. \& Piotrowski, D.( 2019) Differential heterogenesis and the emergence of semiotic function. Semiotica. Issue 230. 